\title{
PRE-COMPACTACIÓN Y ACONDICIONAMIENTO ORGANICO PARA LA RECULTIVACIÓN DE UN SUELO DE TEXTURA GRUESA (TYPIC XEROCHREPTS) DISTURBADO POR EXTRACCIÓN DE ÁRIDOS
}

\section{PRE-COMPACTION AND ORGANIC CONDITIONING FOR THE RECULTIVATION OF A COARSE-TEXTURED SOIL (TYPIC XEROCHREPTS) DISTURBED BY SAND EXTRACTION}

\author{
Oscar Seguel $^{1 *}$, Natalia Rodríguez ${ }^{1}$, Lely Soto ${ }^{1}$, Ian Homer ${ }^{1}$, Carlos Benavides ${ }^{1}$, Manuel \\ Casanova $^{1}$, y Julio Haberland ${ }^{1}$
}

${ }^{1}$ Departamento de Ingeniería y Suelos, Facultad de Ciencias Agronómicas, Universidad de Chile, Avda. Santa Rosa 11.315, La Pintana, Santiago, Chile.

* Autor para correspondencia E-mail: oseguel@uchile.cl

\section{RESUMEN}

La extracción de áridos genera un daño a la estructura del suelo superficial, disminuyendo su capacidad de soporte. Con el objeto de recuperar un suelo agrícola (Typic Xerochrepts, franco arenoso) disturbado por extracción de arenas sub-superficiales, se desarrolló un estudio en la zona central de Chile, en macetas de $20 \mathrm{~L}$, estableciendo tratamientos con dos factores: pre-compactación ( $\sin$ y con dos niveles crecientes) e incorporación de enmienda orgánica (cama broiler en dosis de $0,15,30$ y $60 \mathrm{Mg} \mathrm{ha}^{-1}$ ). Se evaluó el efecto de los tratamientos sobre las propiedades físicas del suelo (densidad aparente, distribución de tamaño de poros, resistencia mecánica, estabilidad de agregados, repelencia al agua) y el rendimiento de materia seca y producción de vainas comerciales de un cultivo de poroto verde (Phaseolus vulgaris L.). La reposición del suelo post-extracción de arena sub-superficial generó una condición de alta porosidad gruesa, baja retención de agua y baja capacidad de soporte; la combinación de la enmienda orgánica en dosis menores a $30 \mathrm{Mg} \mathrm{ha}^{-1} \mathrm{y}$ la ausencia de pre-compactación, promueve un asentamiento excesivo del suelo, lo que disminuye los macroporos y aumenta la resistencia mecánica. Sin embargo, la pre-compactación hasta alcanzar niveles de densidad aparente entre 1,05 y $1,15 \mathrm{Mg} \mathrm{m}^{-3}$ y dosis de enmienda de entre 15 y $30 \mathrm{Mg} \mathrm{ha}^{-1}$, favorecen la mejor condición física del suelo, generando una mayor estabilidad de agregados y la máxima producción de materia seca y rendimiento de vainas comerciales.

Palabras clave: Phaseolus vulgaris, recuperación de suelo, pre-compactación, enmienda orgánica, propiedades físicas de suelo.

\section{ABSTRACT}

Subsoil extraction of subsurface sandy layers causes damages to soil structure, decreasing its loading capacity. A study was conducted in central Chile in order to reclaim an agricultural soil (Typic Xerochrepts, sandy loam) disturbed by subsurface sand mining. The study was conducted in pots of $20 \mathrm{~L}$, and treatments with two factors were established: pre-compaction (without and with two increasing levels), and the addition of organic amendment (broiler litter in doses of $0,15,30$ and $60 \mathrm{Mg} \mathrm{ha}^{-1}$ ). The effect of the treatments was evaluated on soil physical properties (bulk density, pore size distribution, mechanical strength, aggregate stability and water repellency), dry matter yield and pod production of green beans (Phaseolus vulgaris L.). The replenishment of soil after the extraction of subsurface sand generated a condition of high coarse porosity, low water retention and 
low loading capacity. The combination of the organic amendment in doses lower than $30 \mathrm{Mg} \mathrm{ha}^{-1}$ and the absence of pre-compaction promoted excessive soil settlement, decreasing the macroporosity and increasing the mechanical strength. However, pre-compaction until reaching levels of bulk density between 1.05 and $1.15 \mathrm{Mg} \mathrm{m}^{-3}$ and the amendment dose between 15 and $30 \mathrm{Mg} \mathrm{ha}^{-1}$, promoted the best physical soil conditions, resulting in a high aggregate stability, and maximum dry matter production and yield of commercial pods.

Key words: Phaseolus vulgaris, soil reclamation, pre-compaction, organic amendment, soil physical properties.

\section{INTRODUCCIÓN}

La extracción de áridos es una actividad productiva en la cual se retiran materiales geológicos de una determinada área, removiendo el suelo superficial; un caso especialmente importante es la extracción de áridos en terrenos de uso agrícola, donde los materiales de interés se encuentran en estratas sub-superficiales, y para su extracción se necesita remover los horizontes del suelo superficial. Este manejo altera características como la estructura, densidad aparente y capacidad de retención de agua, desarrolladas durante el proceso de pedogénesis, provocando en el suelo una pérdida de funcionalidad como medio físico para los cultivos (Macaya y Gallardo, 2007).

El adecuado crecimiento y desarrollo de cualquier cultivo agrícola depende, entre otras cosas, de la capacidad de arraigamiento que tenga. La porosidad, el agua disponible y la resistencia mecánica del suelo son factores que influyen en la capacidad de anclaje de un cultivo (Krümmelbein y Horn, 2013), todos factores que se ven afectados con la extracción de áridos (Seguel et al., 2014). La recuperación de estos suelos pasa por un proceso de recultivación, el cual consiste en la reposición del material removido, realizando aportes de nutrientes y materia orgánica, además de una adecuada secuencia de cultivos, de manera de favorecer su rápida estructuración (Krümmelbein et al., 2010). La recultivación es un tema poco estudiado en el país, pero en Europa es de gran importancia desde la década del 70 (Kuntze, 1986), e incluso se utiliza como estrategia para la disposición de pasivos ambientales (Zahariev et al., 2014).

La compactación se asocia normalmente a una consecuencia negativa de malos manejos de suelo; sin embargo, en sitios sometidos a extracción de áridos, en que el material edáfico queda excesivamente suelto tras su reposición, la pre-compactación puede ser una herramienta necesaria para acelerar la recuperación del suelo (Seguel et al., 2014). Se entiende la precompactación como una labor de asentamiento de las partículas y/o agregados, a través de una carga mecánica controlada en la superficie del suelo, sin incurrir en un aumento excesivo de la resistencia mecánica de éste (Hartge, 1988).

Junto con la pérdida de la calidad física del suelo, la extracción de áridos genera un efecto de dilución de los nutrientes y de la materia orgánica, ya que la reposición post-extracción no restituye la distribución original de los horizontes del suelo (Macaya y Gallardo, 2007). Según diversos autores (Seguel et al., 2003; Zhang et al., 2005; Abiven et al., 2009) niveles adecuados de materia orgánica en el suelo promueven la formación de macroporos estables $y$, consecuentemente, mejoran la tasa de infiltración, además de facilitar la labranza y permitir una aireación y retención de agua equilibradas. Como complemento, el uso de cultivos acelera el proceso de estructuración, al favorecer los ciclos de secado y humedecimiento, junto con generar importantes aportes de exudados y residuos orgánicos al suelo (Krüemmelbein y Horn, 2013). Además, los cultivos de la familia de las fabáceas elevan la cantidad de nitrógeno $(\mathrm{N})$ en el suelo, debido a la capacidad que tienen de establecer simbiosis con bacterias del género Rhizobium (Faiguenbaum, 2003; Carlsson y Huss-Danell, 2003).

En el presente trabajo se plantea que en suelos con una baja fertilidad natural y una baja retención de agua, como consecuencia de la extracción de áridos, la labor de pre-compactación será más efectiva si se complementa con otras medidas (aplicación de enmiendas orgánicas y uso de cultivos de la familia Fabaceae) con miras a una adecuada recultivación. Trabajos previos se han orientado a habilitar suelos de textura gruesa post-extracción de áridos mediante el uso de enmiendas orgánicas (Macaya y Gallardo, 2007) o mediante labores de pre-compactación (Seguel et al., 2014) por separado, no utilizándose en conjunto ni relacionándolas con el cultivo a establecer. Por lo anterior, el objetivo de este estudio fue evaluar el efecto de la densificación artificial y la incorporación de una enmienda orgánica (cama de broiler) sobre las propiedades físicas del suelo y el desarrollo del cultivo de poroto verde en un ensayo realizado en macetas. 


\section{MATERIALES Y MÉTODOS}

El estudio se realizó en la Estación Experimental Agronómica Germán Greve Silva, Comuna de Maipú, en la Región Metropolitana de Chile (3328` S; 7050` W) durante la temporada 2009. Se utilizó un suelo disturbado (SD) proveniente del sector de extracción de áridos de la Estación Experimental, el que corresponde a la Serie Rinconada de Lo Vial, miembro de la Familia franca gruesa, mixta, térmica de los Typic Xerochrepts, de origen aluvial, generado a partir de depósitos del río Mapocho (CIREN, 1996). El suelo original presenta clases texturales franco arenosas a areno francosas en profundidad, con estructura de bloques subangulares finos y medios débiles en superficie a suelto en profundidad; los colores varían de pardo grisáceo oscuro a pardo oscuro en el matiz $10 \mathrm{YR}$, y se reconoce una secuencia de horizontes $A p-B_{1}-B_{2}-$ $\mathrm{B}_{3}-2 \mathrm{C}$. Bajo los $80 \mathrm{~cm}$ de profundidad, en la zona en que se genera la discontinuidad (horizonte $2 \mathrm{C}$ ) la clase textural es arenosa y, como consecuencia de la extracción de la arena, el suelo superficial queda totalmente mezclado y sin estructura. El análisis previo del SD permitió determinar contenidos de arena, limo y arcilla de 86,$0 ; 7,8 ; \mathrm{y}$ $6,2 \%$, respectivamente.

La enmienda orgánica correspondió a cama de broiler (CB) proveniente de la localidad de Santo Domingo, Región de Valparaíso, que se encontraba en estado fresco, sin estabilizar.

Como unidad experimental se utilizaron contenedores plásticos de $45 \mathrm{~cm}$ de altura y 30 $\mathrm{cm}$ de diámetro $(20 \mathrm{~L})$. Los contenedores fueron perforados en su base para permitir el drenaje del agua de riego, rellenándose con distintas mezclas suelo-enmienda y manteniéndose en un invernadero con ventilación libre. Inicialmente, la CB poseía una alta conductividad eléctrica $\left(\mathrm{CE}=18,8 \mathrm{dS} \mathrm{m}^{-1}\right)$ y alta relación nitrato:amonio (1:10, con valores de 365 y $3598 \mathrm{mg} \mathrm{kg}^{-1}$, respectivamente), por lo que se generó un período de estabilización de dos meses, en el cual se realizaron lavados frecuentes de las mezclas dentro de los contenedores, permitiendo alcanzar valores promedio de $\mathrm{CE}$ menores a 4,2 $\mathrm{dS} \mathrm{m}^{-1} ; 96$ $\mathrm{mg} \mathrm{kg}^{-1}$ de nitrato y $8 \mathrm{mg} \mathrm{kg}^{-1}$ de amonio.

Se realizó un diseño de bloques completos al azar con estructura factorial 3 × 4 (3 niveles de compactación y 4 niveles de materia orgánica) con 3 repeticiones por tratamiento. Los tratamientos consideraron la incorporación de $\mathrm{CB}$ en dosis de $0,15,30$ y $60 \mathrm{Mg}$ ha $^{-1}$ (designados como $\mathrm{O}_{0^{\prime}}$ $\mathrm{O}_{15^{\prime}}, \mathrm{O}_{30} \mathrm{y} \mathrm{O}_{60^{\prime}}$ respectivamente) y niveles de precompactación $\mathrm{P}_{0^{\prime}} \mathrm{P}_{1}$ y $\mathrm{P}_{2}$ (Tabla 1). El nivel de precompactación $\mathrm{P}_{0}$ (sin compactar) fue la resultante del asentamiento natural de las partículas de suelo posterior a un riego, aplicando una carga de $5 \mathrm{~cm}$ de altura de agua, mientras que los niveles de pre-compactación $\mathrm{P}_{1}$ (media) y $\mathrm{P}_{2}$ (alta) se realizaron mediante la aplicación de una fuerza estándar a través de un disco de acero $(7,5 \mathrm{~kg})$, el cual se dejó caer 3 y 5 veces, respectivamente, desde una altura de $1 \mathrm{~m}$ sobre el suelo a capacidad de campo durante el relleno de las macetas (en capas de $10 \mathrm{~cm}$ de espesor).

Se estableció un riego por goteo, con cuatro salidas múltiples distribuidas en la maceta a partir de un gotero de $4 \mathrm{~L} \mathrm{~h}^{-1}$. El seguimiento del contenido de agua se realizó mediante la determinación de la masa de las macetas, regándolas cuando en promedio habían perdido el $50 \%$ del agua aprovechable. Se aplicó un tiempo único de riego, cercano a una hora cada cuatro días al inicio del ensayo, y aumentando la frecuencia cada tres días una vez que el cultivo desarrolló mayor biomasa, manteniendo los tratamientos cercanos a capacidad de campo. Se realizó una fertilización de corrección (Faiguenbaum, 2003) con muriato de potasio y superfosfato triple para satisfacer los requerimientos del cultivo de poroto, aportando $70 \mathrm{~kg} \mathrm{ha}^{-1}$ de $\mathrm{P}$ y $100 \mathrm{~kg} \mathrm{ha}^{-1} \mathrm{de}$ K. Por último, se realizó la siembra de Phaseolus vulgaris L., variedad Magnum, el día 31 de enero de 2009, estableciendo 4 plantas por maceta, en

Tabla 1. Tratamientos con 3 niveles de pre-compactación $(\mathrm{P})$ y 4 niveles de enmienda orgánica $(\mathrm{O})$ aportada como cama de broiler (CB). $P_{i}$ es el nivel de pre-compactación $(0,1$ y 2$)$ y $\mathrm{O}_{\mathrm{i}}$ es el nivel de enmienda orgánica $\left(0,15,30\right.$ y $\left.60 \mathrm{Mg} \mathrm{ha}^{-1}\right)$.

Table 1. Treatments with 3 levels of pre-compaction $(\mathrm{P})$ and 4 levels of organic amendment $(\mathrm{O})$ supplied as broiler litter $(C B)$. $P_{i}$ is the pre-compaction level $(0,1$ and 2$)$ and $\mathrm{O}_{\mathrm{i}}$ is the organic amendment level $\left(0,15,30\right.$ and $\left.60 \mathrm{Mg} \mathrm{ha}^{-1}\right)$.

\begin{tabular}{|c|c|c|c|c|c|}
\hline \multirow[b]{2}{*}{ Pre-compactación $(P)$} & \multicolumn{5}{|c|}{ Dosis de CB (O) } \\
\hline & & 0 & $\begin{array}{c}\mathrm{Mg} \mathrm{ha}^{-1} \\
15\end{array}$ & 30 & 60 \\
\hline Sin pre-compactación & 0 & $\mathrm{P}_{0} \mathrm{O}_{0}$ & $\mathrm{P}_{0} \mathrm{O}_{15}$ & $\mathrm{P}_{0} \mathrm{O}_{30}$ & $\mathrm{P}_{0} \mathrm{O}_{60}$ \\
\hline Pre-compactación media & 1 & $\mathrm{P}_{1} \mathrm{O}_{0}$ & $\mathrm{P}_{1} \mathrm{O}_{15}^{13}$ & $\mathrm{P}_{1} \mathrm{O}_{30}$ & $\mathrm{P}_{1} \mathrm{O}_{60}$ \\
\hline Pre-compactación alta & 2 & $\mathrm{P}_{2} \mathrm{O}_{0}$ & $\mathrm{P}_{2} \mathrm{O}_{15}$ & $\mathrm{P}_{2} \mathrm{O}_{30}$ & $\mathrm{P}_{2} \mathrm{O}_{60}$ \\
\hline
\end{tabular}


una única hilera y distanciadas a $6 \mathrm{~cm}$, siendo las 2 plantas centrales las utilizadas para las distintas mediciones.

Antes de establecer el ensayo, se realizó la prueba de Proctor (Das, 1997) para evaluar la máxima densidad aparente posible de obtener respecto a la dosis de enmienda orgánica.

Se realizaron evaluaciones en dos tiempos de desarrollo del cultivo, la etapa de botón floral se denominó tiempo 1 (T1, 23 de marzo) y la etapa de cosecha se denominó tiempo 2 (T2, 30 de abril). En ambos tiempos se caracterizó el sistema poroso con muestras no disturbadas, utilizando cilindros de $6 \mathrm{~cm}$ de diámetro y $5 \mathrm{~cm}$ de altura, con los cuales se tomaron muestras a 2 profundidades (020 y 20-40 cm), midiendo la retención de agua y la distribución de tamaño de poros mediante cama de arena y olla a presión, ejerciendo tensiones de 2, 60, 100 y $330 \mathrm{hPa}$; finalmente se utilizó un plato de presión para determinar la retención de agua a $15.000 \mathrm{hPa}$ (Sandoval et al., 2012). A partir de la curva de retención de agua se determinó la distribución de tamaño de poros según Hartge y Horn (2009). Con la muestra seca a estufa $\left(105^{\circ} \mathrm{C}\right)$ se determinó la densidad aparente $(\mathrm{Da})$ por el método del cilindro (Sandoval et al., 2012). En el tiempo T2 se midió el índice $\mathrm{R}$ de repelencia al agua (Hallett y Young, 1999) con muestras equilibradas a $-330 \mathrm{hPa}$, el cual se basa en la medición de la sortividad de etanol y agua. De acuerdo a Hallet et al. (2001), valores de $R<2$ corresponden a suelos hidrófilos, con $2 \leq R \leq 4$ se tiene suelos con repelencia sub-crítica y $R>4$ denotan suelos hidrófobos.

Dado que la condición inicial disturbada no permitió medir la estabilidad de agregados al tiempo T1, esta se midió en el tiempo T2 por tamizaje en seco y húmedo (Hartge y Horn, 2009) en una muestra compuesta por cada unidad experimental, que incluyó la profundidad total del suelo $(0-40 \mathrm{~cm})$. También en el tiempo T2 se midió la resistencia mecánica mediante penetrómetro de cono (Koolen y Kuipers, 1983) en cuatro puntos por maceta y en rangos de profundidad de 0-2, 2-5, 5-10 y 10-20 cm. La evaluación se hizo 24 horas después de un riego, de manera de tener el suelo en una tensión mátrica equivalente a capacidad de campo (Hartge y Horn, 2009).

Los parámetros del cultivo consideraron las dos plantas centrales de cada maceta. En el primer periodo (T1) se cuantificó la materia seca del cultivo en estado de botón floral, mediante secado a $70^{\circ} \mathrm{C}$ hasta masa constante. En el segundo periodo (T2), se evaluó además la cantidad y masa de vainas de desecho por plantas (curvas y deformes) y el rendimiento promedio de vainas comerciales por tratamiento $\left(\mathrm{Mg} \mathrm{ha}^{-1}\right)$.
Debido a que se realizaron dos evaluaciones en el tiempo, se consideró el doble de macetas, totalizando 72 unidades experimentales. Para la curva Proctor se realizó un ajuste parabólico, de manera de determinar el contenido de agua con el cual se logra la máxima densidad aparente. Para cada una de las propiedades evaluadas, se realizó un análisis de varianza (ANDEVA, $\alpha \leq 0,05$ ) con el programa JMP 8 (Ramírez y Ramírez, 2009) y cuando hubo diferencias significativas se realizó una prueba de comparación múltiple (LSD, $\alpha \leq$ $0,05)$.

\section{RESULTADOS Y DISCUSIÓN}

\section{Propiedades físicas de suelo}

Prueba de Proctor y densidad aparente. Se evaluó la susceptibilidad a la compactación del suelo para las distintas dosis de enmienda orgánica (CB). A modo de ejemplo, en la Fig. 1 se observa el resultado para la menor y la máxima dosis de CB de los tratamientos.

El coeficiente de curvatura resultante va disminuyendo en relación al aumento de $\mathrm{CB}(25,1$; 12,$6 ; 10,9$ y 7,7 , respectivamente para $\mathrm{O}_{0}, \mathrm{O}_{15}, \mathrm{O}_{30}$ $\left.\mathrm{y}_{60}\right)$, lo que evidencia el efecto de la enmienda orgánica sobre el comportamiento mecánico del suelo, alcanzando valores de densidad aparente máximos de $1,69 \mathrm{Mg} \mathrm{m}^{-3}$ para $\mathrm{O}_{0}$ a $1,47 \mathrm{Mg} \mathrm{m}^{-3}$ para $\mathrm{O}_{60}$, asociados a contenidos de agua de 18 y $19 \%$ (base gravimétrica), respectivamente. Es decir, al aumentar la dosis de enmienda existe una mayor retención de agua y una menor compactabilidad del suelo. En el caso de suelos de clase textural gruesa, la acción de la materia orgánica ocurre entre las partículas, promoviendo un aumento del comportamiento elástico del suelo (Zhang et al., 2005).

A pesar de ser un sustrato areno francoso, los suelos presentaron valores de densidad aparente (Da) (Tabla 2) en los rangos típicos de suelos de texturas finas (Sandoval et al., 2012), muy por debajo de los valores máximos determinados por la prueba de Proctor.

En términos generales, los niveles de Da no fueron restrictivos para el cultivo, ya que no se verifican valores elevados que pudieran reducir el crecimiento y el desarrollo de raíces (Casanova et al., 2008). Al periodo de botón floral (T1), con la dosis más alta de enmienda y la menor pre-compactación $\left(\mathrm{P}_{0} \mathrm{O}_{60}\right)$ se observó la Da más baja, tanto en superficie como en profundidad, diferenciándose estadísticamente de los tratamientos con dosis de enmienda menores. El nivel de pre-compactación siguió una lógica sólo en las muestras de $20-40 \mathrm{~cm}$ de profundidad y en dosis mayores a $30 \mathrm{Mg} \mathrm{ha}^{-1} \mathrm{de} \mathrm{CB}$, destacando al agua como un agente de ordenamiento de 


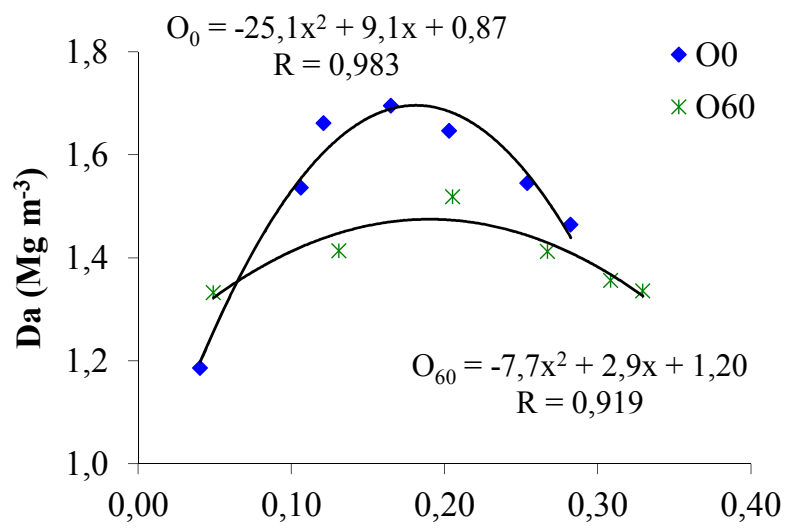

Contenido de agua $\left(\mathrm{g} \mathrm{g}^{-1}\right)$

Fig. 1. Prueba de Proctor para los tratamientos con aplicación de cama de broiler (CB) en dosis de 0 y $60 \mathrm{Mg} \mathrm{ha}^{-1}$. Ajustes presentan el $\mathrm{R}$ significativo al 95\% $(\alpha<0,05)$.

Fig. 1. Proctor test for treatments with organic amendment (CB) at doses of 0 and $60 \mathrm{Mg} \mathrm{ha}^{-1}$. Adjusts show the $\mathrm{R}$ value at $95 \%(\alpha<0.05)$.

Tabla 2. Densidad aparente $\left(\mathrm{Mg} \mathrm{m}^{-3}\right.$, promedio $\left.\pm \mathrm{DS}\right)$ para dos profundidades $(0-20 \mathrm{y} 20-40 \mathrm{~cm})$ y dos tiempos de evaluación (T1 y T2). $P_{\mathrm{i}}$ es el nivel de pre-compactación y $\mathrm{O}_{\mathrm{i}}$ es el nivel de enmienda.

Table 2. Bulk density $\left(\mathrm{Mg} \mathrm{m}^{-3}\right.$, average $\left.\pm \mathrm{SD}\right)$ at two depths $(0-20$ and $20-40 \mathrm{~cm})$ and two times (T1 and T2) of evaluation. $P_{i}$ is the pre-compaction level and $\mathrm{O}_{\mathrm{i}}$ is the amendment level.

\begin{tabular}{|c|c|c|c|c|}
\hline \multirow{2}{*}{$\begin{array}{l}\text { Dosis enmienda } \\
\text { Pre-compactación }\end{array}$} & $\mathrm{O}_{0}$ & $\mathrm{O}_{15}$ & $\mathrm{O}_{30}$ & $\mathrm{O}_{60}$ \\
\hline & \multicolumn{4}{|c|}{ Densidad aparente $\left(\mathrm{Mg} \mathrm{m}^{-3}\right)$} \\
\hline & \multicolumn{4}{|c|}{ 0-20 cm (T1) } \\
\hline $\mathbf{P}_{0}$ & $1,25 \pm 0,06 \mathrm{ab}$ & $1,20 \pm 0,02 a b$ & $1,07 \pm 0,10 \mathrm{bc}$ & $0,95 \pm 0,12 \mathrm{c}$ \\
\hline $\mathbf{P}_{1}$ & $1,26 \pm 0,05 a b$ & $1,10 \pm 0,02 a b c$ & $1,07 \pm 0,06 \mathrm{bc}$ & $1,11 \pm 0,09 a b c$ \\
\hline \multirow[t]{2}{*}{$\mathbf{P}_{2}$} & $1,28 \pm 0,07 \mathrm{a}$ & $1,21 \pm 0,04 \mathrm{ab}$ & $1,25 \pm 0,11 \mathrm{ab}$ & $1,07 \pm 0,03 \mathrm{bc}$ \\
\hline & \multicolumn{4}{|c|}{ 20-40 cm (T1) } \\
\hline $\mathbf{P}_{0}$ & $1,34 \pm 0,07 \mathrm{a}$ & $1,16 \pm 0,07 \mathrm{abc}$ & $1,06 \pm 0,06 \mathrm{~cd}$ & $0,94 \pm 0,05 \mathrm{~d}$ \\
\hline $\mathbf{P}_{1}$ & $1,20 \pm 0,03 \mathrm{abc}$ & $1,13 \pm 0,06 \mathrm{bcd}$ & $1,19 \pm 0,02 a b c$ & $1,08 \pm 0,06 \mathrm{~cd}$ \\
\hline \multirow[t]{2}{*}{$\mathbf{P}_{2}^{1}$} & $1,28 \pm 0,04 \mathrm{ab}$ & $1,22 \pm 0,03 \mathrm{abc}$ & $1,23 \pm 0,01 \mathrm{abc}$ & $1,11 \pm 0,09 \mathrm{bcd}$ \\
\hline & \multicolumn{4}{|c|}{ 0-20 cm (T2) } \\
\hline $\mathbf{P}_{0}$ & $1,14 \pm 0,01 \mathrm{abcd}$ & $1,01 \pm 0,06 \quad \mathrm{~cd}$ & $1,16 \pm 0,07$ abcd & $0,95 \pm 0,13 \mathrm{~d}$ \\
\hline$P_{1}$ & $1,24 \pm 0,14 \mathrm{abc}$ & $1,13 \pm 0,03 \mathrm{abcd}$ & $1,19 \pm 0,10 \mathrm{abc}$ & $1,08 \pm 0,03 \mathrm{bcd}$ \\
\hline \multirow[t]{2}{*}{$\mathbf{P}_{2}$} & $1,33 \pm 0,03 \mathrm{a}$ & $1,28 \pm 0,08 \mathrm{ab}$ & $1,20 \pm 0,10 \mathrm{abc}$ & $1,10 \pm 0,02 \mathrm{bcd}$ \\
\hline & \multicolumn{4}{|c|}{$20-40 \mathrm{~cm}(\mathrm{~T} 2)$} \\
\hline $\mathbf{P}_{0}$ & $1,23 \pm 0,03 a b$ & $0,97 \pm 0,07 \mathrm{c}$ & $0,99 \pm 0,09 c$ & $1,02 \pm 0,07 \mathrm{c}$ \\
\hline $\mathbf{P}_{1}$ & $1,23 \pm 0,12 \mathrm{ab}$ & $1,09 \pm 0,06 \mathrm{bc}$ & $1,13 \pm 0,07 \mathrm{bc}$ & $1,16 \pm 0,03 a b c$ \\
\hline $\mathbf{P}_{2}$ & $1,34 \pm 0,06 \mathrm{a}$ & $1,29 \pm 0,04 \mathrm{ab}$ & $1,33 \pm 0,08 \mathrm{a}$ & $1,24 \pm 0,02 \mathrm{ab}$ \\
\hline
\end{tabular}

Existió interacción $\mathrm{PxO}$, por lo que letras distintas reflejan diferencias estadísticas significativas entre tratamientos a un mismo tiempo y profundidad (LSD, $\alpha<0,05)$.

partículas más efectivo que la densificación artificial (Semmel et al., 1990).

Al final del ensayo (T2) se da la lógica en cuanto a un aumento de la Da a medida que aumenta la pre-compactación en ambas profundidades, lo que se asocia a un mayor desarrollo de raíces, que junto a los procesos de mojamiento y secado favorecen un ordenamiento de partículas en forma inversamente proporcional al nivel de densificación; se genera así la mayor soltura 
de suelo en las situaciones en que las raíces encontraron la menor resistencia mecánica $\left(\mathrm{P}_{0}\right)$, tal como lo muestran Sellés et al. (2012) con aplicaciones de materia orgánica en huertos de vid.

En relación a los niveles máximos de Da posibles de alcanzar según la prueba de Proctor, los tratamientos sin enmienda orgánica $\left(\mathrm{O}_{0}\right)$ alcanzaron niveles de compactación entre $70 \mathrm{y}$ $80 \%$, mientras que para la dosis mayor de $\mathrm{CB}\left(\mathrm{O}_{60}\right)$ el nivel de compactación se ubicó entre el 65 y el $76 \%$, aunque en una situación $\left(\mathrm{P}_{2} \mathrm{O}_{60}\right)$ se superó el $80 \%$. Estos niveles responden a la baja estabilidad del material arenoso incluso con altas dosis de $\mathrm{MO}$, destacando la labor descompactadora de la raíz del cultivo al tiempo T2 (Baginsky et al., 2010).

Distribución de tamaño de poros y retención de agua. A partir de la curva característica evaluada en ambos periodos (T1 y T2) y a las dos profundidades de suelo, se clasificaron los poros por tamaño, siendo relevantes los resultados en macroporosidad (Tablas 3 y 4 ).

En el tiempo T1 en superficie, se observa que las mayores macroporosidades se presentan en aquellos tratamientos con un grado ligero de pre-compactación $\left(\mathrm{P}_{1}\right)$. La acción conjunta de las tensiones externas (pre-compactación artificial) e internas (ciclos de secado y humectación) en el caso de $\mathrm{P}_{1}$ resulta en un sistema poroso estable, cumpliendo con la teoría de la tensión efectiva (Seguel y Horn, 2005) que favorece la estabilidad mecánica; tal hecho fue demostrado por Zúñiga et al. (2015) en sistemas pratenses, donde el pisoteo previo generó una estabilidad porosa sostenida en el tiempo. En profundidad existe la tendencia a aumentar la macroporosidad hasta la

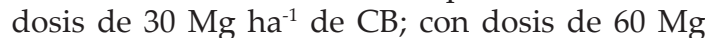
ha $^{-1}$ los macroporos disminuyen, demostrando la inestabilidad que generan las aplicaciones excesivas de materia orgánica (Seguel et al., 2003). A la cosecha (T2), las muestras superficiales $(0-20 \mathrm{~cm})$ se homogenizaron en términos porosos, no presentando diferencias estadísticas significativas. En cambio, en profundidad $(20-40 \mathrm{~cm})$ se mantiene la tendencia del tiempo T1, con mayor abundancia de macroporos en las dosis intermedias de enmienda orgánica y con un nivel de pre-compactación ligero.

Al comparar el comportamiento de la macroporosidad entre ambos tiempos, se observa una disminución, tanto en superficie $(0-20 \mathrm{~cm})$ como en profundidad $(20-40 \mathrm{~cm})$, debido al aumento de contracción por secado (Seguel y Horn, 2005). Aun así los macroporos (> 50

Tabla 3. Poros de drenaje rápido $(\mathrm{PDR},>50 \mu \mathrm{m}$, promedio $\pm \mathrm{DS})$ en los tiempos $\mathrm{T} 1$ y T2. $\mathrm{P}_{\mathrm{i}}$ es el nivel de pre-compactación y $\mathrm{O}_{\mathrm{i}}$ es el nivel de enmienda.

Table 3. Fast drainage pores (PDR, $>50 \mu \mathrm{m}$, average \pm SD) at times $\mathrm{T} 1$ and $\mathrm{T} 2 . \mathrm{P}_{\mathrm{i}}$ is the pre-compaction level and $\mathrm{O}_{\mathrm{i}}$ is the amendment level.

\begin{tabular}{|c|c|c|c|c|}
\hline Dosis enmienda & $\mathrm{O}_{0}$ & $\mathrm{O}_{15}$ & $\mathrm{O}_{30}$ & $\mathbf{O}_{60}$ \\
\hline Pre-compactación & \multicolumn{4}{|c|}{ PDR (\%) } \\
\hline & \multicolumn{4}{|c|}{ 0-20 cm (T1) } \\
\hline $\mathbf{P}_{0}$ & $16,2 \pm 1,8 \quad b$ & $20,3 \pm 0,7 \mathrm{ab}$ & $23,7 \pm 2,6 a b$ & $25,7 \pm 2,7 \mathrm{a}$ \\
\hline $\mathbf{P}_{1}$ & $22,0 \pm 2,1 \mathrm{ab}$ & $26,7 \pm 3,2 \mathrm{a}$ & $26,5 \pm 2,9$ a & $24,9 \pm 4,2 \mathrm{ab}$ \\
\hline $\mathbf{P}_{2}$ & $20,0 \pm 0,9 a b$ & $21,0 \pm 1,5 \mathrm{ab}$ & $18,8 \pm 1,9 a b$ & $21,5 \pm 1,7 \mathrm{ab}$ \\
\hline & \multicolumn{4}{|c|}{$20-40 \mathrm{~cm} \mathrm{(T1)}$} \\
\hline $\mathbf{P}_{0}$ & $20,4 \pm 1,4 \mathrm{ab}$ & $24,0 \pm 2,2 \mathrm{ab}$ & $24,0 \pm 2,5 \mathrm{ab}$ & $22,3 \pm 2,3 a b$ \\
\hline $\mathbf{P}_{1}$ & $22,4 \pm 1,5 \mathrm{ab}$ & $21,3 \pm 2,1 \mathrm{ab}$ & $25,6 \pm 0,9 a$ & $22,4 \pm 3,1 \mathrm{ab}$ \\
\hline $\mathbf{P}_{2}$ & $18,4 \pm 1,8 \mathrm{ab}$ & $26,0 \pm 1,8 \mathrm{a}$ & $24,8 \pm 1,9 \mathrm{ab}$ & $15,9 \pm 1,6 b$ \\
\hline & \multicolumn{4}{|c|}{ 0-20 cm (T2) } \\
\hline $\mathbf{P}_{0}$ & $17,0 \pm 3,2 \mathrm{a}$ & $22,2 \pm 3,8 \mathrm{a}$ & $17,8 \pm 3,6$ a & $22,9 \pm 4,6 a$ \\
\hline $\mathbf{P}_{1}$ & $17,3 \pm 3,8$ a & $22,2 \pm 4,3 \mathrm{a}$ & $19,5 \pm 2,9 a$ & $18,5 \pm 2,1 \mathrm{a}$ \\
\hline $\mathbf{P}_{2}$ & $15,7 \pm 3,3$ a & $16,3 \pm 3,1 \mathrm{a}$ & $16,8 \pm 3,0$ a & $20,6 \pm 2,5 \mathrm{a}$ \\
\hline & \multicolumn{4}{|c|}{$20-40 \mathrm{~cm}(\mathrm{~T} 2)$} \\
\hline $\mathbf{P}_{0}$ & $19,9 \pm 3,1 \mathrm{abc}$ & $18,4 \pm 3,2 \mathrm{abc}$ & $24,6 \pm 3,4 a b$ & $20,6 \pm 3,1 a b$ \\
\hline $\mathbf{P}_{1}$ & $18,9 \pm 3,4 a b c$ & $27,4 \pm 3,8 \mathrm{a}$ & $27,7 \pm 4,1 \mathrm{a}$ & $19,0 \pm 4,6 \mathrm{abc}$ \\
\hline $\mathbf{P}_{2}$ & $20,2 \pm 3,0 a b c$ & $20,9 \pm 3,3$ abc & $16,3 \pm 2,8 \mathrm{bc}$ & $13,4 \pm 2,8 \mathrm{c}$ \\
\hline
\end{tabular}

Existió interacción $\mathrm{PxO}$, por lo que letras distintas reflejan diferencias estadísticas significativas entre tratamientos a un mismo tiempo y profundidad (LSD, $\alpha<0,05)$. 
$\mu \mathrm{m})$, para todas las muestras, se encuentran en cantidad abundante de acuerdo a Horn y Fleige (2009)

Los poros de drenaje lento (PDL) corresponden a un tamaño de poro que pierde agua por la atracción gravitacional a una velocidad más lenta que los poros de drenaje rápido (PDR), de tal forma que la raíz de los vegetales podría absorber dicha agua, considerándosele dentro del rango de agua fácilmente disponible (Horn y Fleige, 2009). Es común que este tamaño de poros se presente en menor proporción respecto a los poros más gruesos (Tabla 3), pues corresponderían a estados intermedios de re-estructuración posterior a un estado degradado del suelo (Seguel et al., 2013). Aunque sin diferencias estadísticas significativas en superficie para el primer periodo evaluado, los resultados mantienen la tendencia vista para los PDR, en la que la dosis intermedia de CB y de pre-compactación se conjugan en forma ideal para el enraizamiento, generando un sistema poroso altamente estable, el que incluso puede aumentar producto de la renovación del sistema radical del cultivo (Seguel et al., 2013).

En cuanto a los poros de agua útil, solo se observaron diferencias estadísticas en el tiempo T1 en superficie $(0-20 \mathrm{~cm})$, donde los tratamientos con elevadas dosis de CB y grados de pre-compactación $\left(\mathrm{P}_{1}\right.$ y $\left.\mathrm{P}_{2}\right)$ presentaron el mayor porcentaje de este tipo de poros $(25,6 \%)$, en contraste con $\mathrm{P}_{1} \mathrm{O}_{15}$, que presentó el menor nivel $(15,7 \%)$. Al respecto, se confirma que la incorporación de enmiendas orgánicas en suelos de texturas gruesas aumenta el contenido de poros con un tamaño menor a $10 \mu \mathrm{m}$ (Mtanbanengwe et al., 2004), favoreciendo la retención de agua útil para la planta. Si bien los resultados presentaron una alta variabilidad y no fue posible en el tiempo T2 detectar diferencias estadísticas significativas en rangos de contenido de agua de 14 a $28 \%$ (base volumen), de acuerdo a Reynolds et al. (2009) todos los casos corresponden a contenidos de agua útil en rangos de bueno a ideal para el crecimiento de los cultivos.

Repelencia al agua y estabilidad. La repelencia al agua, entendida como la reducción del mojamiento causado por la presencia de cubiertas hidrofóbicas sobre las partículas minerales, es generalmente de naturaleza orgánica (Hallet, 2008). No obstante que un exceso de hidrofobia es negativo en el suelo, ya que dificulta el proceso de infiltración y genera una mala distribución del agua en el perfil, es necesario un ligero comportamiento hidrófobo para mantener una buena estabilidad al agua (Goebel et al., 2005).

Tabla 4. Poros de drenaje lento (PDL, 10-50 $\mu \mathrm{m}$, promedio \pm DS) en los tiempos T1 y T2. $P_{\mathrm{i}}$ es el nivel de pre-compactación y $\mathrm{O}_{\mathrm{i}}$ es el nivel de enmienda.

Table 4. Slow drainage pores (PDL, 10-50 $\mu \mathrm{m}$, average $\pm \mathrm{DS}$ ) at times $\mathrm{T} 1$ and $\mathrm{T} 2 . \mathrm{P}_{\mathrm{i}}$ is the precompaction level and $\mathrm{O}_{\mathrm{i}}$ is the amendment level.

\begin{tabular}{|c|c|c|c|c|}
\hline Dosis enmienda & $\mathrm{O}_{0}$ & $\mathrm{O}_{15}$ & $\mathrm{O}_{30}$ & $\mathrm{O}_{60}$ \\
\hline Pre-compactación & \multicolumn{4}{|c|}{ PDL (\%) } \\
\hline & \multicolumn{4}{|c|}{ 0-20 cm (T1) } \\
\hline $\mathbf{P}_{0}$ & $12,1 \pm 4,5 \mathrm{a}$ & $11,6 \pm 4,3$ a & $11,8 \pm 4,1 \mathrm{a}$ & $7,4 \pm 4,0 \mathrm{a}$ \\
\hline $\mathbf{P}_{1}$ & $15,2 \pm 6,8$ a & $10,9 \pm 3,9 a$ & $12,1 \pm 3,9 \mathrm{a}$ & $9,9 \pm 3,2 \mathrm{a}$ \\
\hline \multirow[t]{2}{*}{$\mathbf{P}_{2}$} & $6,9 \pm 4,2 \mathrm{a}$ & $13,3 \pm 4,4 \mathrm{a}$ & $12,1 \pm 3,7 \mathrm{a}$ & $7,7 \pm 4,3 \mathrm{a}$ \\
\hline & \multicolumn{4}{|c|}{ 20-40 cm (T1) } \\
\hline $\mathbf{P}_{0}$ & $15,5 \pm 4,1 \mathrm{ab}$ & $18,2 \pm 3,9 a$ & $15,6 \pm 3,4 \mathrm{ab}$ & $14,0 \pm 3,1 \mathrm{ab}$ \\
\hline$P_{1}$ & $14,4 \pm 4,0 \mathrm{ab}$ & $13,9 \pm 3,6 \mathrm{ab}$ & $17,2 \pm 3,6 \mathrm{ab}$ & $14,8 \pm 3,7 \mathrm{ab}$ \\
\hline \multirow[t]{2}{*}{$\mathbf{P}_{2}$} & $12,9 \pm 3,7 \mathrm{ab}$ & $20,3 \pm 5,7 a$ & $19,4 \pm 3,6$ a & $9,3 \pm 3,0 b$ \\
\hline & \multicolumn{4}{|c|}{$0-20 \mathrm{~cm}(\mathrm{~T} 2)$} \\
\hline$P_{0}$ & $9,3 \pm 2,3 \mathrm{ab}$ & $8,8 \pm 1,8 \mathrm{ab}$ & $7,1 \pm 2,2 \mathrm{ab}$ & $7,7 \pm 2,1 \mathrm{ab}$ \\
\hline$P_{1}$ & $10,0 \pm 2,8 \mathrm{ab}$ & $7,3 \pm 1,9 \mathrm{ab}$ & $8,3 \pm 2,7 \mathrm{ab}$ & $6,1 \pm 1,5 b$ \\
\hline \multirow[t]{2}{*}{$\mathbf{P}_{2}$} & $9,6 \pm 2,0 \mathrm{ab}$ & $10,2 \pm 3,1 \mathrm{a}$ & $9,3 \pm 2,9 \mathrm{ab}$ & $7,5 \pm 1,9 \mathrm{ab}$ \\
\hline & \multicolumn{4}{|c|}{$20-40 \mathrm{~cm}(\mathrm{~T} 2)$} \\
\hline$P_{0}$ & $13,1 \pm 3,9 \mathrm{ab}$ & $10,1 \pm 3,0 \mathrm{ab}$ & $14,1 \pm 3,4 \mathrm{ab}$ & $12,9 \pm 2,7 \mathrm{ab}$ \\
\hline$P_{1}$ & $11,9 \pm 2,8 \mathrm{ab}$ & $17,0 \pm 3,8 \mathrm{a}$ & $14,4 \pm 3,0 \mathrm{ab}$ & $11,5 \pm 2,8 \mathrm{ab}$ \\
\hline $\mathbf{P}_{2}$ & $13,8 \pm 3,2 \mathrm{ab}$ & $11,2 \pm 3,3 \mathrm{ab}$ & $11,9 \pm 2,9 \mathrm{ab}$ & $9,1 \pm 2,2 b$ \\
\hline
\end{tabular}

Existió interacción $\mathrm{PxO}$, por lo que letras distintas reflejan diferencias estadísticas significativas entre tratamientos a un mismo tiempo y profundidad (LSD, $\alpha<0,05)$. 
El índice de repelencia ( $R$, adimensional) no presentó diferencias estadísticas significativas y los valores variaron entre 1,7 y 3,5, lo que califica a los suelos como ligeramente hidrofílicos a ligeramente hidrofóbicos (Hallet et al., 2001).

A pesar de no presentar diferencias estadísticas significativas, los tratamientos con una mayor dosis de CB presentaron los mayores valores de $R$, lo cual indica un mayor grado de hidrofobia. Los bajos niveles de $\mathrm{MO}(<1,2 \%)$ en los tratamientos sin enmienda $\left(\mathrm{O}_{0}\right)$ generaron un comportamiento hidrófilo, el que implicará una entrada violenta del agua al suelo, provocando el colapso estructural. Esta situación se revierte con la pre-compactación, la cual afecta la sortividad del agua por la menor disponibilidad de poros de conducción (Lipiec et al., 2009). No existió una correlación entre el contenido de MO y $R$, ni entre éste y la estabilidad de agregados, dejando de manifiesto la compleja relación órgano-mineral en el proceso de estructuración, en la que la hidrofobia depende también del tipo de MO, su ubicación, la actividad biológica, la superficie específica del suelo y el contenido de agua (Hallet, 2008).

Los procesos de humedecimiento del suelo, como son las precipitaciones o riegos, generan inestabilidad o destrucción de las uniones órganominerales, provocando la dispersión (Chen et al., 2000). Mediante la variación del diámetro (VD) de los agregados entre un tamizaje en seco y otro en húmedo, es posible determinar la tendencia del suelo a ser dispersado, de manera tal que un suelo inestable presentará mayores valores de VD. En la Tabla 5 se detalla el valor de la VD para cada tratamiento en una muestra compuesta de las dos profundidades, comprobándose que existe una fuerte relación entre la dosis de enmienda y la estabilidad estructural, con diferencias significativas respecto al testigo $\left(\mathrm{P}_{0} \mathrm{O}_{0}\right)$, incluso con bajas dosis de MO.

A pesar que la menor estabilidad de agregados se observó en el tratamiento $\mathrm{P}_{0} \mathrm{O}_{0} \mathrm{y}$ la mayor estabilidad de agregados se da en $\mathrm{P}_{2} \mathrm{O}_{60}$ se aprecia que la VD no depende del nivel de precompactación, por lo que se puede concluir que ésta no favoreció el contacto materia orgánicapartículas minerales. Los resultados indican que el suelo, posterior a la extracción de áridos, presentará dispersión de un $80 \%$ promedio de sus agregados si se prescinde de una enmienda orgánica, resultando crucial el efecto de ésta sobre la recuperación de la calidad del suelo.

Seguel et al. (2011), estudiando un suelo franco arenoso del norte de Chile y degradado por pisoteo, obtuvieron niveles de dispersión similares (cercanos al 80\%) y determinaron que al cabo de tres años de manejo con cultivos se pueden alcanzar niveles de dispersión menores al $40 \%$. Aun así los resultados pueden ser mejores si se utiliza estiércol sobre un suelo con contenidos de arcilla mayores al del presente estudio, ya que ambos factores son determinantes en los procesos de estructuración (Seguel et al., 2013).

Resistencia mecánica. La resistencia mecánica del suelo dice relación con la capacidad de éste para soportar cargas externas (Zhang et al., 2005) así como la facilidad de enraizamiento de los cultivos (Busscher y Bauer, 2003). La resistencia depende, entre otros factores, de la textura, el tipo de arcilla, el contenido y tipo de materia orgánica, la agregación y el contenido de agua del suelo (Horn, 2004). Los resultados de la evaluación de resistencia mecánica realizada $24 \mathrm{~h}$ después de un riego se presenta en la Tabla 6. Como el potencial mátrico es el mismo para todos los tratamientos, las diferencias dependen exclusivamente del contenido de materia orgánica y del nivel de precompactación.

Como era de esperar, se cumplió la respuesta físico-mecánica de un suelo sometido a precompactación, en el sentido que al aumentar esta última aumenta la resistencia, especialmente con dosis elevadas de enmienda orgánica. Claramente

Tabla 5. Variación del diámetro de los agregados (VD 70\%, promedio \pm DS) entre un tamizaje en seco y otro en húmedo al tiempo T2. $\mathrm{P}_{\mathrm{i}}$ es el nivel de pre-compactación y $\mathrm{O}_{\mathrm{i}}$ es el nivel de enmienda.

Table 5. Aggregate diameter variation (VD 70\%, average \pm SD) between dry and wet sieving at time T2. $P_{i}$ is the pre-compaction level and $O_{i}$ is the amendment level.

\begin{tabular}{ccccc}
\hline Dosis enmienda & \multicolumn{1}{c}{$\mathbf{O}_{0}$} & $\mathbf{O}_{15}$ & $\mathbf{O}_{30}$ & $\mathbf{O}_{60}$ \\
\cline { 2 - 5 } Pre-compactación & \multicolumn{4}{c}{ VD 70 (\%) } \\
\hline $\mathbf{P}_{0}$ & $84,3 \pm 8,7 \mathrm{a}$ & $42,3 \pm 19,0 \mathrm{bc}$ & $17,2 \pm 2,1 \mathrm{~cd}$ & $19,3 \pm 9,6 \mathrm{~cd}$ \\
$\mathbf{P}_{1}$ & $79,6 \pm 3,0 \mathrm{a}$ & $67,4 \pm 6,5 \mathrm{ab}$ & $17,6 \pm 5,8 \mathrm{~cd}$ & $24,3 \pm 9,0 \mathrm{~cd}$ \\
$\mathbf{P}_{2}$ & $79,9 \pm 5,7 \mathrm{a}$ & $43,9 \pm 19,5 \mathrm{bc}$ & $15,8 \pm 9,9 \mathrm{~cd}$ & $5,9 \pm 2,6 \mathrm{~d}$ \\
\hline
\end{tabular}

Letras distintas implican diferencias estadísticas significativas entre los tratamientos (LSD, $\alpha<0,05$ ). 
Tabla 6. Resistencia mecánica $\left(\mathrm{kPa}\right.$, promedio \pm DS) del suelo para distintas profundidades. $\mathrm{P}_{\mathrm{i}}$ es el nivel de pre-compactación y $\mathrm{O}_{\mathrm{i}}$ es el nivel de enmienda.

Table 6. Mechanical strength of soil $(\mathrm{kPa}$, average $\pm \mathrm{SD})$ at different depths. $P_{\mathrm{i}}$ is the pre-compaction level and $\mathrm{O}_{\mathrm{i}}$ is the amendment level.

\begin{tabular}{|c|c|c|c|c|}
\hline Dosis enmienda & $\mathrm{O}_{0}$ & $\mathrm{O}_{15}$ & $\mathrm{O}_{30}$ & $\mathrm{O}_{60}$ \\
\hline Pre-compactación & \multicolumn{4}{|c|}{ Resistencia mecánica (kPa) } \\
\hline & \multicolumn{4}{|c|}{$0-2 \mathrm{~cm}$} \\
\hline $\mathbf{P}_{0}$ & $138 \pm 54 \mathrm{a}$ & $71 \pm 38 \mathrm{ab}$ & $32 \pm 7 \quad b$ & $22 \pm 5 b$ \\
\hline$P_{1}$ & $71 \pm 11 \mathrm{ab}$ & $66 \pm 23 \mathrm{ab}$ & $95 \pm 22 \mathrm{ab}$ & $71 \pm 28 \mathrm{ab}$ \\
\hline \multirow[t]{2}{*}{$\mathbf{P}_{2}$} & $129 \pm 27 \mathrm{a}$ & $94 \pm 51 \mathrm{ab}$ & $126 \pm 14 \mathrm{a}$ & $119 \pm 59 \mathrm{a}$ \\
\hline & \multicolumn{4}{|c|}{$2-5 \mathrm{~cm}$} \\
\hline$P_{0}$ & $166 \pm 38 \mathrm{ab}$ & $72 \pm 30 \mathrm{cde}$ & $17 \pm 7$ e & $53 \pm 6$ de \\
\hline $\mathbf{P}_{1}$ & $138 \pm 14 \mathrm{abc}$ & $148 \pm 49 \mathrm{ab}$ & $148 \pm 36 \mathrm{ab}$ & $114 \pm 51 \mathrm{bcc}$ \\
\hline \multirow[t]{2}{*}{$\mathbf{P}_{2}$} & $200 \pm 7 \quad a$ & $143 \pm 45 \mathrm{ab}$ & $185 \pm 5$ a & $140 \pm 13 \mathrm{abc}$ \\
\hline & \multicolumn{4}{|c|}{$5-10 \mathrm{~cm}$} \\
\hline $\mathbf{P}_{0}$ & $233 \pm 29 \mathrm{ab}$ & $94 \pm 35$ ef & $40 \pm 20 \mathrm{f}$ & $99 \pm 21$ ef \\
\hline$P_{1}$ & $165 \pm 5 \mathrm{~cd}$ & $152 \pm 16$ de & $132 \pm 53 \mathrm{de}$ & $136 \pm 22 \mathrm{de}$ \\
\hline \multirow[t]{2}{*}{$\mathbf{P}_{2}$} & $260 \pm 10 \mathrm{ab}$ & $165 \pm 24 \mathrm{~cd}$ & $280 \pm 10 \mathrm{a}$ & $215 \pm 35 b c$ \\
\hline & \multicolumn{4}{|c|}{$10-20 \mathrm{~cm}$} \\
\hline $\mathbf{P}_{0}$ & $250 \pm 70 b c$ & $53 \pm 6$ e & $80 \pm 10 \mathrm{de}$ & $77 \pm 25 \mathrm{de}$ \\
\hline$P_{1}$ & $203 \pm 55 c$ & $220 \pm 20 \mathrm{bc}$ & $133 \pm 30 \mathrm{~d}$ & $107 \pm 15$ de \\
\hline $\mathbf{P}_{2}$ & $315 \pm 15 a$ & $240 \pm 10 \mathrm{bc}$ & $315 \pm 15 a$ & $270 \pm 30 \mathrm{ab}$ \\
\hline
\end{tabular}

Existió interacción $\mathrm{PxO}$, por lo que letras distintas implican diferencias estadísticas significativas entre los tratamientos a una misma profundidad (LSD, $\alpha<0,05)$.

el tratamiento $\mathrm{P}_{2} \mathrm{O}_{0}$ presentó los mayores valores de resistencia, mientras que $\mathrm{P}_{0} \mathrm{O}_{60}$ mantuvo una baja resistencia en todo el perfil, ayudado por la estructura fibrosa de la enmienda (Zhang et al., 2005); sin embargo, no se cumple con claridad la secuencia descendente de resistencia a medida que aumenta la dosis de enmienda. La CB utilizada incluía materiales fibrosos y gruesos (hasta $5 \mathrm{~cm}$ ) de difícil descomposición, lo que pudo mantener en el perfil una condición de alta heterogeneidad, generando los resultados variables de la Tabla 6 . Los tratamientos $\mathrm{O}_{0}$ presentaron, para un mismo nivel de pre-compactación, un aumento gradual de resistencia en profundidad, debido a la masa acumulada del material superficial (Hartge, 1988; Horn et al., 2007); esta lógica no se dio claramente en los tratamientos que tuvieron aplicación de $\mathrm{CB}$, por cuanto el material grueso de la enmienda genera heterogeneidades a través del perfil.

Taylor y Brar (1991) señalan un valor crítico (entre 150 y $600 \mathrm{kPa}$ ) a partir del cual se comienza a afectar la penetración radical, valor que depende del cultivo y sus condiciones ambientales (nutrición, agua, aire). Sin embargo, la fuerza axial que es capaz de ejercer una raíz le permite desarrollarse en suelos con hasta $2.000 \mathrm{kPa}$ (Horn and Fleige, 2009), por lo que la condición final del ensayo no sería restrictiva para el enraizamiento de los cultivos.

\section{Desarrollo y producción del cultivo}

Considerando que el poroto verde se siembra en la zona central en primavera (de noviembre a enero), este ensayo se inició tardíamente (fines de enero) debido a la alta CE generada por la adición de la enmienda, lo que se solucionó con lavados sucesivos a través del riego. Así, la Tabla 7 presenta el crecimiento en términos de materia seca, a los dos tiempos de desarrollo del cultivo (T1 y T2), además de las vainas de desecho y el rendimiento comercial del cultivo.

Los primeros estados de crecimiento del cultivo, hasta botón floral (tiempo T1), no mostraron mayores diferencias en materia seca (MS) entre los tratamientos. En el tiempo T2 se observaron diferencias significativas en el crecimiento del cultivo, con el mayor valor en el tratamiento $\mathrm{P}_{1} \mathrm{O}_{15}$ y el más bajo valor de crecimiento en $\mathrm{P}_{2} \mathrm{O}_{60}$. Cuando se realizaron aplicaciones de $\mathrm{MO}$ en altas dosis (> $30 \mathrm{Mg} \mathrm{ha}^{-1}$ ) la pre-compactación máxima (P2) resultó adversa para el desarrollo del cultivo, por cuanto disminuyó el diámetro de los poros, generando condiciones de excesiva humedad y baja aireación y temperatura, de acuerdo a lo que señala Reynolds et al. (2009) como condición ideal para el desarrollo de los cultivos.

Diversos autores señalan un comportamiento de parábola invertida al relacionar la MS producida y la densidad aparente (Lindstrom y Voorhees, 
Tabla 7. Rendimiento de materia seca aérea $\left(\mathrm{g} \mathrm{planta}^{-1}\right)$ en los tiempos T1 y T2; cantidad de vainas de desecho, $\mathrm{y}$ rendimiento de vainas útiles $\left(\mathrm{Mg} \mathrm{ha}^{-1}\right)$. Valores promedio $\pm \mathrm{DS}$ de seis plantas por tratamiento. $P_{i}$ es el nivel de pre-compactación y $\mathrm{O}_{\mathrm{i}}$ es el nivel de enmienda.

Table 7. Dry matter yield (g plant ${ }^{-1}$ ) at time T1 and T2; amount of non-commercial pods, and yield of useful pods $\left(\mathrm{Mg} \mathrm{ha}^{-1}\right)$. Average $\pm \mathrm{DS}$ of six plants per treatment. $P_{i}$ is the pre-compaction level and $\mathrm{O}_{\mathrm{i}}$ is the amendment level.

\begin{tabular}{|c|c|c|c|c|}
\hline Dosis enmienda & $\mathrm{O}_{0}$ & $\mathrm{O}_{15}$ & $\mathrm{O}_{30}$ & $\mathrm{O}_{60}$ \\
\hline Pre-compactación & \multicolumn{4}{|c|}{ Materia seca T1 (g) } \\
\hline $\mathbf{P}_{0}$ & $11,1 \pm 0,6 \mathrm{a}$ & $18,9 \pm 2,9 \mathrm{a}$ & $21,3 \pm 6,8 \mathrm{a}$ & $19,1 \pm 6,8 \mathrm{a}$ \\
\hline $\mathbf{P}_{1}$ & $12,9 \pm 4,3 \mathrm{a}$ & $20,1 \pm 3,0 \mathrm{a}$ & $21,0 \pm 7,5 \mathrm{a}$ & $12,2 \pm 4,7 \mathrm{a}$ \\
\hline \multirow[t]{2}{*}{$\mathbf{P}_{2}$} & $9,8 \pm 1,4 \mathrm{a}$ & $21,0 \pm 3,9 \mathrm{a}$ & $16,4 \pm 1,3 \mathrm{a}$ & $9,0 \pm 1,1 \mathrm{a}$ \\
\hline & \multicolumn{4}{|c|}{ Materia seca T2 (g) } \\
\hline$P_{0}$ & $19,7 \pm 7,8 \mathrm{bc}$ & $31,3 \pm 7,6 a b c$ & $31,4 \pm 7,9 \mathrm{abc}$ & $23,8 \pm 7,8 a b c$ \\
\hline $\mathbf{P}_{1}$ & $28,8 \pm 8,5 \mathrm{abc}$ & $41,6 \pm 9,8 \mathrm{a}$ & $27,5 \pm 8,1 \mathrm{abc}$ & $22,6 \pm 7,7 \mathrm{bc}$ \\
\hline \multirow[t]{2}{*}{$\mathbf{P}_{2}$} & $20,8 \pm 7,9 \mathrm{bc}$ & $35,5 \pm 8,8 \mathrm{ab}$ & $25,3 \pm 8,5 \mathrm{abc}$ & $15,0 \pm 6,9 \mathrm{c}$ \\
\hline & \multicolumn{4}{|c|}{ Cantidad (\#) vainas de desecho } \\
\hline $\mathbf{P}_{0}$ & $13 \pm 10 b$ & $38 \pm 12 a$ & $22 \pm 3 \mathrm{ab}$ & $22 \pm 10 a b$ \\
\hline $\mathbf{P}_{1}$ & $26 \pm 9 a b$ & $32 \pm 4 \quad a b$ & $24 \pm 5 \mathrm{ab}$ & $16 \pm 2 a b$ \\
\hline \multirow[t]{2}{*}{$\mathbf{P}_{2}$} & $12 \pm 4 \quad b$ & $23 \pm 14 a b$ & $17 \pm 4 \mathrm{ab}$ & $10 \pm 6 \quad b$ \\
\hline & \multicolumn{4}{|c|}{ Rendimiento comercial $\left(\mathrm{Mg} \mathrm{ha}^{-1}\right)$} \\
\hline $\mathbf{P}_{0}$ & $3,28 \pm 2,91 \mathrm{a}$ & $4,09 \pm 4,99 \mathrm{ab}$ & $5,20 \pm 2,15 a b$ & $6,00 \pm 2,41 \mathrm{ab}$ \\
\hline$P_{1}$ & $7,57 \pm 1,78 \mathrm{ab}$ & $11,92 \pm 1,03 b$ & $4,74 \pm 1,83 a b$ & $7,30 \pm 2,17 \mathrm{ab}$ \\
\hline $\mathbf{P}_{2}$ & $5,06 \pm 1,35 \mathrm{ab}$ & $4,70 \pm 4,66 \mathrm{ab}$ & $6,94 \pm 4,49 a b$ & $4,24 \pm 1,86 \mathrm{ab}$ \\
\hline
\end{tabular}

Letras distintas implican diferencias estadísticas entre los tratamientos (LSD, $\alpha<0,05$ ).

1994; Kuth y Reintam, 2001). Dicho resultado depende del equilibrio agua/aire influenciado por aspectos climáticos, de manera tal que dosis altas de MO pueden generar un exceso de retención de agua, afectando el desarrollo del cultivo en forma directa con cada nivel de densificación (Hakansson, 2005). Con dosis bajas de CB $\left(<15 \mathrm{Mg}\right.$ ha- $^{-}$ $\left.{ }^{1}\right)$, la pre-compactación ligera $\left(\mathrm{P}_{1}\right)$ logró el mejor contacto suelo-raíz-agua, generando los mayores rendimientos, tal como lo observaron Seguel et al. (2014) en un ensayo de campo con el mismo suelo de este estudio.

Considerando un cultivo establecido con 290.000 plantas ha $^{-1}$, se genera una producción de MS de 1,5 y 4,0 $\mathrm{Mg}^{-1}$ para los tratamientos $\mathrm{P}_{2} \mathrm{O}_{60}$ y $\mathrm{P}_{1} \mathrm{O}_{15}$, respectivamente. Manivannan et al. (2009) evaluaron la producción de MS de poroto al cabo de 60 días de crecimiento en un suelo franco arenoso, determinando niveles de 0,9 a 1,4 Mg ha ${ }^{-1}$ para tratamientos con aplicación de vermicompost (5 $\mathrm{Mg} \mathrm{ha}^{-1}$ ) y vermicompost con fertilización mineral $(50 \%$ de dosis de vemicompost y 10:40:20 $\mathrm{kg} \mathrm{ha}^{-1}$ de NPK). Por su parte, Castellaro y Squella (2006) plantean que las praderas mediterráneas de Chile central, bajo un adecuado suministro nutricional, pueden generar producciones de MS cercanas a $4 \mathrm{Mg} \mathrm{ha}^{-1}$ en condiciones de secano. Es decir, los resultados obtenidos en el presente estudio apuntan a que al generar una adecuada relación agua/aire/ resistencia mecánica en el suelo recultivado, la producción de MS puede alcanzar niveles óptimos, e incluso ser similar a la producida por una pradera.

Las vainas producidas por las plantas fueron separadas en categorías de vainas útiles (rectas y con más de $10 \mathrm{~cm}$ de largo) y vainas de desecho (menos de $10 \mathrm{~cm}$ de largo y/o curvas). No hubo una relación clara entre las condiciones de suelo y la cantidad de vainas sin calidad comercial (Tabla 7), cuya presencia podría deberse a un efecto de competencia entre plantas. Al respecto, Getachew et al. (2015) informaron una relación directa entre vainas rectas y el espaciamiento de las plantas, resultando crucial la distancia sobre la hilera para evitar deformaciones. Existe la tendencia de una menor producción de vainas de desecho en los tratamientos extremos $\left(\mathrm{P}_{0} \mathrm{O}_{0}\right.$ y $\left.\mathrm{P}_{2} \mathrm{O}_{60}\right)$, aunque la alta variabilidad de los resultados impidió tener diferencias significativas en cuanto a su masa, la cual varió entre 5 y 30 g por planta (datos no incluidos).

Con respecto a la estimación del rendimiento de vainas productivas en cosecha (en base a 290.000 plantas ha $^{-1}$ ), los resultados de la Tabla 7 no incluyen aquellas vainas curvas o de bajo calibre, las que son descartadas para la industria y generalmente desechadas por las máquinas 
cosechadoras.

Para una plantación comercial, se esperan rendimientos de entre 6-14 $\mathrm{Mg} \mathrm{ha}^{-1}$ en suelos francos (Urzúa et al., 2003). Manivannan et al. (2009) obtuvieron rendimientos de 7,1 a $11,3 \mathrm{Mg}$ ha $^{-1}$ cuando se realizan aplicaciones de vermicompost o mezclas de vermicompost con fertilizantes sintéticos, muy por encima del testigo $\left(4,75 \mathrm{Mg} \mathrm{ha}^{-1}\right)$ sin enmiendas. En el presente ensayo, los rendimientos estuvieron dentro de lo esperado, a pesar de los niveles de salinidad presente en el sustrato y a la fecha tardía de siembra. Además se comprobó un comportamiento lógico, en función de los niveles de enmienda y de precompactación, con un rendimiento alto para el tratamiento con labores de manejo intermedio $\left(\mathrm{P}_{1} \mathrm{O}_{15}\right)$. Se puede apreciar que en $\mathrm{P}_{0} \mathrm{O}_{0}$ se verificaron los menores rendimientos, cumpliéndose la condición desfavorable para el buen desarrollo del cultivo; por otra parte, las dosis excesivas de materia orgánica tampoco aseguran una condición favorable para la productividad agrícola.

Finalmente, para asegurar la conservación de la capa fértil del suelo post extracción de áridos y mejorar el proceso de recultivación, es necesaria la educación y legislación ambiental, proporcionando conocimientos sitio-específicos a los usuarios sobre el impacto ambiental de estas faenas degradantes de los suelos.

\section{CONCLUSIONES}

El suelo disturbado, producto de la extracción de arenas subsuperficiales del perfil, puede ser mejorado mediante las labores de precompactación de mediana intensidad, en conjunto con la incorporación de una enmienda orgánica en base a cama de broiler, lo que resulta en una adecuada relación sólidos/agua/aire para el enraizamiento de los cultivos.

Valores de densidad aparente de $1,1 \mathrm{Mg} \mathrm{m}^{-3}$ y dosis de $15 \mathrm{Mg}^{-1}$ de enmienda orgánica, promovieron altos niveles de macroporosidad con niveles intermedios de estabilidad de agregados y de resistencia mecánica. Esta condición de suelo aseguró los mayores rendimientos en el cultivo de poroto, tanto de materia seca como de vainas comerciales, lo que lo proyecta como una buena alternativa para recuperar suelos degradados con expectativas de buenos rendimientos comerciales.

\section{LITERATURA CITADA}

Abiven, S., S. Menasseri, and C. Chenu. 2009. The effects of organic inputs over time on soil aggregate stability - A literature analysis. Soil Biology and Biochemistry 41:1-12.
Baginsky, C., O. Seguel, y A. Contreras. 2010. Impacto en la utilización de cultivos y enmiendas orgánicas sobre la funcionalidad del suelo. Serie Ciencias Agronómicas № 17. Universidad de Chile, Santiago, Chile.

Burés, S. 1997. Sustratos. Ediciones Agrotécnicas SL, Madrid, España.

Busscher, W. J., and P. J. Bauer. 2003. Soil strength, cotton root growth and line yield in a southeastern USA coastal loamy sand. Soil and Tillage Research 74:151-159.

Carlsson, G., and K. Huss-Danell. 2003. Nitrogen fixation in perennial forage legumes in the field. Plant and Soil 253: 353-372.

Casanova, M., O. Seguel, J. Haberland, y C. Kremer. 2008. Propiedades físicas: Indicadores de calidad y salud de suelos. Revista Antumapu 6(1-2):32-34.

Castellaro, G., y F. Squella. 2006. Modelo simple de simulación para la estimación del crecimiento, fenología y balance hídrico de praderas anuales de clima mediterráneo. Agricultura Técnica (Chile) 66(3):271-282.

Chen, C., Y. Le Bissonnais, and D. Arrouays. 2000. Organic matter influence on clay wettability and soil aggregate stability. Soil Science Society of America Journal 64:1479-1486.

CIREN, 1996. Estudio Agrológico Región Metropolitana. Descripción de suelos, materiales y símbolos. Publicación CIREN $\mathrm{N}^{\circ}$ 115. Centro de Información de Recursos Naturales (CIREN), Santiago, Chile.

Das, B. M. 1997. Soil mechanics laboratory manual. Engineering Press, Austin, Texas, USA.

Faiguenbaum, H. 2003. Labranza, siembra y producción de los principales cultivos de Chile. Ograma S.A., Santiago, Chile.

Getachew E., A. Mohammed, and A. Tesfaye. 2015. Impact of sowing date and plant spacing on yield, quality and disease incidence of Snap bean (Phaseolus vulgaris L.) varieties at Jimma Southwestern, Ethiopia. Global Advanced Research Journal of Educational Research and Review 4(5):081-089.

Goebelt, M.-O., J. Bachmann, S. Woche, and W. R. Fischer. 2005. Soil wettability, aggregate stability, and the decomposition of soil organic matter. Geoderma 128:80-93.

Hakansson, I. 2005. Machinery-induced compaction of arable soils. Incidencesconsequences-counter-measures. Reports from the Division of Soil Management No. 109. Swedish University of Agricultural Sciences, Department of Soil Sciences, Uppsala, Sweden. 
Hallet, P.D. 2008. A brief overview of the causes, impacts and amelioration of soil water repellency - a review. Soil and Water Research 3(1):521-529.

Hallet, P.D., T. Baumgartl, and J. M. Young. 2001. Subcritical water repellency of aggregates from a range of soil management practices. Soil Science Society of America Journal. 65:184-190.

Hallet, P.D., and I. M. Young. 1999. Changes to water repellence of soil aggregates caused by substrate-induced microbial activity. European Journal of Soil Science 50:35-40.

Hartge, K.H. 1988. The reference base for compaction state of soils. p. 73-77. In J. Drescher, Horn, R. and de Boodt, M. (eds.). Impact of water and external forces on soil structure. Catena Supplement 11, Cremlingen, Germany.

Hartge, K.H., und R. Horn. 2009. Die physikelische Untersuchung von Böden: Praxis, Messmethodem, Auswestung. E. Schweizerbast Verlagsbuchhandlung, Stuttgart, Germany.

Horn, R. 2004. Time dependence of soil mechanical properties and pore functions in arable soils. Soil Science Society of America Journal 68:1131-1137.

Horn, R., K. R. Hartge, J. Bachmann, and M. B. Kirkham. 2007. Mechanical stresses in soils assessed from bulk density and penetrationresistance data set. Soil Science Society of America Journal 71(5):1455-1459.

Horn, R., and R. Fleige. 2009. Risk assessment of subsoil compaction for arable soils in Northwest Germany at farm scale. Soil and Tillage Research 102(2):201-208.

Koolen, A.J., and H. Kuipers. 1983. Agricultural soil mechanics. Advances Series in Agricultural Sciences 13. Springer-Verlag, Berlin, Germany.

Kuntze, H. 1986. Soil reclamation, improvement, recultivation and conservation in Germany. Zeitschrift für Pflanzenernaehrung und Bodenkunde 149:500-512.

Kuth, J., and R. Reitam. 2001. The impact of deep rooted plants on the qualities of compacted soils. p. 632-636. In D.E. Stott, Mohtar; R.H. and Steinhardt, G.C. (eds.). Sustaining the Global Farm. Selected papers from the $10^{\text {th }}$ International Soil Conservation Organization Meeting. International Soil Conservation Organization (ISCO), Purdue, Indiana, USA.

Krümmelbein, J., and R. Horn. 2013. Cycle of soil structure. p. 183-198. In J. Krümmelbein, Horn, R. and Pagliai, M. (eds.). Soil degradation. Advances in Geoecology 42. IUSS, Reiskirchen, Germany.
Krümmelbein, J., R. Horn., Th. Raab, O. Bens and R. Hüttl. 2010. Soil physical parameters of a recently established agricultural recultivation site after brown coal mining in Eastern Germany. Soil and Tillage Research 111(1):19-25.

Lindstrom, M.J., and W.B. Voorhees. 1994. Responses of temperate crops in North America to soil compaction. p. 265-286. In B. Soane and van Ouwerkerk, C. (eds.). Soil compaction in crop production. Developments in Agricultural Engineering 11. Elsevier. The Netherlands.

Lipiec, J., A. Wójciga, and R. Horn. 2009. Hydraulic properties of soil aggregates as influenced by compaction. Soil and Tillage Research 103:170-177.

Macaya, C., y M. Gallardo. 2007. Manual de rehabilitación de suelos sometidos a extracción de áridos en zonas agrícolas. Servicio Agrícola y Ganadero, Santiago, Chile.

Manivannan, S., M. Balamurugan, K. Parthasarathi, G. Gunasekaran, and L.S. Ranganathan. 2009. Effect of vermicompost on soil fertility and crop productivity - beans (Phaseolus vulgaris). Journal of Environmental Biology 30(2):275-281.

Mtanbanengwe, F., P. Mapfumo, and $\mathrm{H}$. Kirchmann. 2004. Decomposition of organic matter in soils influenced by the texture and the pore size distribution. p. 261-275. In A. Bationo (ed.). Managing nutrient cycles to sustain soil fertility in sub-Saharan Africa. Academy Sciences Publisher, Nairobi, Kenia.

Ramírez, J. G., and B. S. Ramírez. 2009. Analyzing and interpreting continuous data using JMP: A step by step guide. SAS Institute Inc., Cary, North Carolina,USA.

Reynolds, W., C. Drury, C. Tan, C. Fox, and X. Yang. 2009. Use of indicators and pore volume-function characteristics to quantify soil physical quality. Geoderma 152:252-263.

Sandoval, M., J. Dörner, O. Seguel, J. Cuevas, y D. Riveras. 2012. Métodos de análisis físicos de suelos. Publicación $\mathrm{N}^{\circ} 5$. Universidad de Concepción, Facultad de Agronomía, Departamento de Suelos y Recursos Naturales, Chillán, Chile.

Seguel, O., V. García de Cortázar, y M. Casanova. 2003. Variación en el tiempo de las propiedades físicas de un suelo con adición de enmiendas orgánicas. Agricultura Técnica (Chile) 63(3):287-297.

Seguel, O., and R. Horn. 2005. Structure properties and pore dynamics in aggregate beds due to wetting and drying cycles. Journal of Plant Nutrition and Soil Science 169:221-232. 
Seguel, O., C. Baginsky, A. Contreras, J. Covarrubias, C. González, y M. Sandoval. 2011. Changes in physical properties of a Typic Haplocambid by annual crop culture. Journal of Soil Science and Plant Nutrition 11(1):1-15.

Seguel, O., C. Baginsky, A. Contreras, J.I. Covarrubias, C. González, y L. Poblete. 2013. Physical properties of a fine textured haplocambid after three years of organic matter amendments management. Journal of Soil Science and Plant Nutrition 13(3):690-705.

Seguel, O., I. Cárcamo, M. Casanova, I. Homer, J. Haberland, y C. Benavides. 2014. Precompactación como herramienta para mejorar la relación agua/aire en habilitación de suelos post-extracción de áridos. Agro Sur 42(1):51-62.

Sellés, G., R. Ferreyra, R. Ruiz, R. Ferreyra, y R. Ahumada. 2012. Compactación de suelos y su control: Estudio de casos en el Valle de Aconcagua. Boletín 234. Instituto de Investigaciones Agropecuarias (INIA), Santiago, Chile.

Semmel, H., R. Horn, A.R. Dexter, and D.E. Schulze. 1990. The dynamics of soil aggregate formation and the effect on soil physical properties. Soil Technology 3:113-129.

Sierra, C. 2003. Fertilización de cultivos y frutales en la zona norte. Boletín INIA $N^{\circ} 97$. Instituto de Investigaciones Agropecuarias (INIA), La Serena, Chile.
Steppuhn, H., M. Th. Van Genuchten, and C. M. Grieve. 2005. Root-zone salinity: II. Indices for tolerance in agricultural crops. Crop Science 45:221-232.

Taylor, H.M., and G.S. Brar. 1991. Effect of soil compaction on root development. Soil and Tillage Research 19:111-119.

Urzúa, H., L. Barrales, H. Faiguenbaum, M. Gálvez, R. Ormazábal, y R. Pizarro. 2003. Fertilización nitrogenada de porotos verdes para uso agroindustrial en la zona central de Chile: indicaciones preliminares. Ciencia e Investigación Agraria 30(1):57-60.

Zahariev, A., Sv. Kostadinova, and A. Aladjadjiyan. 2014. Composting municipal waste for soil recultivation in Bulgaria. International Journal of Plant \& Soil Science 3(2):178-185.

Zhang, B., R. Horn, and P. Hallet. 2005. Mechanical resilience of degraded soil amended with organic matter. Soil Science Society of America Journal 69:864-871.

Zúñiga, F., J. Ivelic-Sáez, I. López, D. Huygens, and J. Dörner. 2015. Temporal dynamics of the physical quality of an Andisol under a grazing system subjected to different pasture improvement strategies. Soil and Tillage Research 145:233-241. 